\title{
Iterative probe correction technique for spherical near-field antenna measurements
}

\author{
Laitinen, Tommi; Pivnenko, Sergey; Breinbjerg, Olav
}

Published in:

I E E E Antennas and Wireless Propagation Letters

Link to article, DOI:

10.1109/LAWP.2005.851069

Publication date:

2005

Document Version

Publisher's PDF, also known as Version of record

Link back to DTU Orbit

Citation (APA):

Laitinen, T., Pivnenko, S., \& Breinbjerg, O. (2005). Iterative probe correction technique for spherical near-field antenna measurements. I E E E Antennas and Wireless Propagation Letters, 4, 221-223.

https://doi.org/10.1109/LAWP.2005.851069

\section{General rights}

Copyright and moral rights for the publications made accessible in the public portal are retained by the authors and/or other copyright owners and it is a condition of accessing publications that users recognise and abide by the legal requirements associated with these rights.

- Users may download and print one copy of any publication from the public portal for the purpose of private study or research.

- You may not further distribute the material or use it for any profit-making activity or commercial gain

- You may freely distribute the URL identifying the publication in the public portal 


\title{
Iterative Probe Correction Technique for Spherical Near-Field Antenna Measurements
}

\author{
T. A. Laitinen, S. Pivnenko, Member, IEEE, and O. Breinbjerg, Member, IEEE
}

\begin{abstract}
An iterative probe correction technique is presented to correct for the errors caused by the application of the traditional first-order probe correction for nonideal first-order probes in spherical near-field antenna measurements. The technique may be applied, with certain restrictions, for more general probes as well.
\end{abstract}

Index Terms-Antenna, first-order probe, high-order probe correction, probe correction, spherical wave expansion.

\section{INTRODUCTION}

$\mathbf{T}$ HE probe-corrected spherical near-field measurement is a well-established technique for the characterization of radiation from antennas [1]. It is based on solving for the unknown $\mathrm{Q}$ coefficients $\left(Q_{s m n}\right)$ in the spherical vector wave expansion of the radiated field from the antenna under test (AUT) employing the transmission formula [1, Ch. 3]

$$
\begin{aligned}
& w(r, \chi, \theta, \phi) \\
& \quad=\sum_{n=1}^{N} \sum_{m=-n}^{n} \sum_{s=1}^{2} \sum_{\mu} Q_{s m n} e^{i m \phi} d_{\mu m}^{n}(\theta) e^{i \mu \chi} P_{s \mu n}(k r) .
\end{aligned}
$$

Here, $w$ is the signal at the output of the probe, $(r, \theta, \phi)$ are the standard spherical coordinates, $\chi$ is the probe rotation angle, $N$ is the truncation number for the spherical wave expansion, $e^{i m \phi}$, $d_{\mu m}^{n}(\theta)$, and $e^{i \mu \chi}$ are the rotation coefficients, and $P_{s \mu n}(k r)$ are the probe response constants [1, Ch. 3].

For first-order probes, i.e., for probes with azimuthal spherical mode index restricted to $\mu= \pm 1$, the probe correction can be performed efficiently using the traditional first-order probe correction technique [1, Chs. 3 and 4]. The field radiated by an ideal open-ended circular waveguide probe, excited by the $\mathrm{TE}_{11}$ waveguide mode, contains only the first-order azimuthal spherical modes, and thus this probe is an example of an ideal first-order probe [1, Ch. 5].

In practice, real probes are never ideal first-order probes but possess, due to, e.g., the manufacturing errors, also a minor level of high-order modes. Applying the first-order probe correction for a real probe thus means that a full probe correction is not obtained. The resulting errors may in some cases be acceptable, while in other cases, e.g., where high accuracy is required, unacceptable [2].

The purpose of this letter is to present a simple iterative technique to correct for the errors caused by the application of the first-order probe correction for nonideal first-order probes. It

Manuscript received February 21, 2005; revised April 29, 2005

The authors are with Ørsted.DTU, Electromagnetic Systems, Technical University of Denmark, 2800 Lyngby, Denmark (e-mail: tla@oersted.dtu.dk).

Digital Object Identifier 10.1109/LAWP.2005.851069 is shown by a simulation that a nonideal first-order probe may cause significant errors in the prediction of the directivity, and it is further shown that the presented iterative probe correction technique can be used to correct for these errors.

It is noted, that in [3], though not stated explicitly, the test zone field (TZF) compensation technique includes, in addition to compensation for range reflections and RF leakage from the cables, also compensation for the probe. The iterative probe correction technique presented here may thus be reasonably compared with the TZF compensation technique in case the only extraneous fields present in the TZF [3] are those caused by the high-order spherical modes of the probe. In this case, these two techniques essentially differ from each other by the separate calibration measurement they require. The complexity of the TZF calibration measurement is determined by the size of the AUT, whereas the complexity of the probe pattern calibration required by the iterative probe correction technique presented here is determined by the size of the probe. Since probes are generally smaller than AUTs the probe calibration measurement is easier to perform than the TZF calibration measurement.

\section{THEORY}

The probe signal of the transmission formula may be written as a sum of the desired signal, i.e., the signal due to the $\mu= \pm 1$ modes of the probe, and the error signal, i.e., the signal due to the $\mu \neq \pm 1$ modes of the probe

$$
w(r, \chi, \theta, \phi)=w_{d}(r, \chi, \theta, \phi)+w_{e}(r, \chi, \theta, \phi)
$$

where the desired and the error signals, respectively, are

$$
\begin{aligned}
& w_{d}(r, \chi, \theta, \phi) \\
& =\sum_{n=1}^{N} \sum_{m=-n}^{n} \sum_{s=1}^{2} \sum_{\mu= \pm 1} Q_{s m n} e^{i m \phi} d_{\mu m}^{n}(\theta) e^{i \mu \chi} P_{s \mu n}(k r) \\
& w_{e}(r, \chi, \theta, \phi) \\
& =\sum_{n=1}^{N} \sum_{m=-n}^{n} \sum_{s=1}^{2} \sum_{\mu \neq \pm 1} Q_{s m n} e^{i m \phi} d_{\mu m}^{n}(\theta) e^{i \mu \chi} P_{s \mu n}(k r) .
\end{aligned}
$$

For simplicity, we now write (4) in the form of a function as $w=$ $f\left\{Q_{s m n}\right\}$, and equation (3) in the form of an inverse operation as $Q=\Phi^{-1}\{w\}$. The inverse operator $\Phi^{-1}$ corresponds to the first-order probe correction [1, Ch. 4]. If we assume that $w_{e}(r, \chi, \theta, \phi)$ is zero, this inverse operation provides the correct values for the $\mathrm{Q}$ coefficients. The iterative probe correction may now be expressed simply as the following two equations:

$$
\begin{aligned}
Q_{s m n}^{(l)} & =\Phi^{-1}\left\{w-w_{e}^{(l)}\right\} \\
w_{e}^{(l+1)} & =f\left\{Q_{s m n}^{(l)}\right\}
\end{aligned}
$$




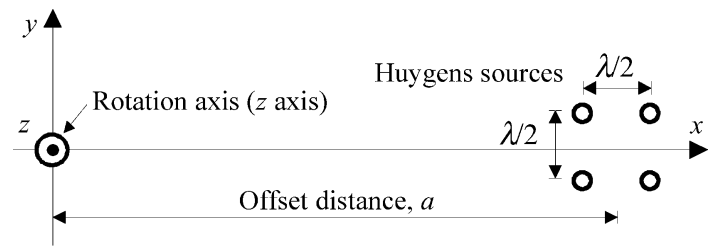

Fig. 1. AUT model.

where $l=0 \ldots L$, and $L$ is the number of iterations to be chosen appropriately. The value of $L$ depends on the probe properties, the radiated field of the AUT, and the measurement distance. The initial value for the error signal $w_{e}^{(0)}=0$ may be used, and $Q_{s m n}^{(0)}$ thus are the $\mathrm{Q}$ coefficients determined by the traditional first-order probe correction. Equation (6) determines the error signal, and this requires the probe response constants to be known for $\mu \neq \pm 1$, as obtained from the probe calibration.

The above-presented formulation assumes that a single-port probe is used to sample the field for $\chi=0^{\circ}$ and $90^{\circ}$ so the probe must be turned $90^{\circ}$ during the measurement. Yet, the technique may be formulated for a two-port probe as well, so that the timemultiplexed electronic switching between the two ports during the measurement is sufficient and the probe rotation thus not required.

\section{SimULATIONS}

A series of simulations for the AUT model shown in Fig. 1 is performed to verify the iterative probe correction technique. The AUT model consists of four y-polarized Huygens sources excited in phase, so that the maximum radiation of each source is in the $+\mathrm{z}$ axis direction. The center point of the AUT is located at an offset distance $a$ from the azimuthal rotation axis of the spherical measurement system. In the simulation series, the offset distance $a$ varies from 2 to $8 \lambda$ in steps of $2 \lambda$.

The measured radiation pattern of a real probe is used in the simulation. In this work a low-quality open-ended circular waveguide is used to represent a nonideal first-order probe. The normalized $\mu$-mode power spectrum [1, Ch. 6], [2] of this probe is presented in Fig. 2. Since all the power is not concentrated in modes $\mu= \pm 1$, this clearly illustrates that the probe is not an ideal first-order probe.

First the correct Q coefficients of the AUT are determined from the known tangential electric field [1, Ch. 2-4]. The signal received by the probe is then calculated in the sampling directions at the measurement distance $r$ using the transmission formula (1). In the simulation series the measurement distance $r$ varies from $a+2 \lambda$ to $10 \lambda$ in steps of $1 \lambda$. From the calculated received signals of the probe, the iterative probe correction technique is finally used to calculate the Q coefficients of the AUT and then the corresponding far field.

\section{RESULTS}

The iterative probe correction technique has been observed to work well in each of the 16 afore-mentioned simulation cases, i.e., accurate predictions of the far fields are obtained. The correct operation of the technique was verified by investigating the following convergences with number of iterations $L: 1$ ) the

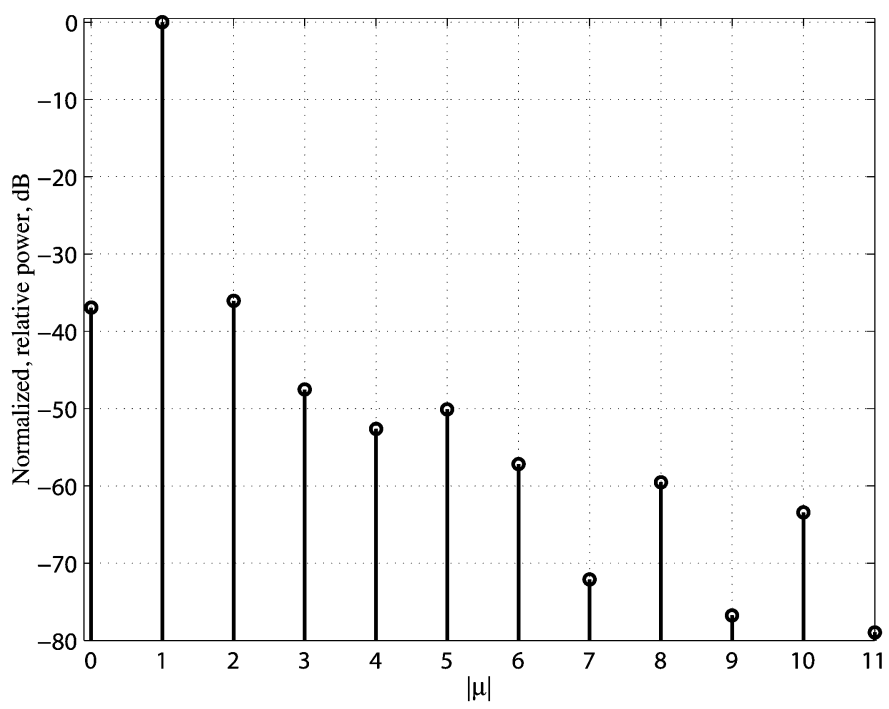

Fig. 2. Normalized $\mu$-mode power spectrum of the radiated field of a nonideal open-ended circular waveguide.

convergence of two successive iterations of the predicted field, $E_{L}-E_{L-1}$, and 2) the convergence of the predicted field $E_{L}$ and the correct field $E$. The first convergence will show that the iterations can be truncated with appropriate criterion, whereas the second convergence will show that the predicted field $E_{L}$ converges to the correct field $E$. The results for the above-mentioned simulation cases show, that for the measurement distances $r \geq a+3 \lambda$, and for the number of iterations $L=3$, the relative power of the difference between two successive predicted fields is always less than $-100 \mathrm{~dB}$. Also, the relative power of the difference between the predicted and correct fields is always less than $-140 \mathrm{~dB}$. In conclusion, a fast and correct convergence is reached in all cases that correspond to the separation distance between the minimum spheres of the AUT and the probe greater than $1 \lambda$.

As a representative example, the simulation result of the AUT model located on the $4 \lambda$ offset distance, for the $10 \lambda$ measurement distance, is presented in Fig. 3, where the errors between the predicted and the correct directivities in the $x-z$ plane are shown for the case without iteration, i.e., the traditional first-order probe correction, and for the case with one iteration, i.e., for $L=1$. The result clearly shows that a low-quality open-ended circular waveguide causes an error in the predicted directivity of the AUT, which may be unacceptable in some cases [4]. The result also shows that the error is almost completely removed by using the iterative probe correction technique with only one iteration.

\section{CONCLUSION}

In this letter, an iterative probe correction technique has been presented for correcting the errors caused by the application of the first-order probe correction for a nonideal first-order probe in the spherical near-field antenna measurements. The technique has been tested by a series of simulated spherical near-field antenna measurements, and shown to work. Using the iterative technique, instead of the traditional first-order probe correction technique [1, Ch. 4], allows the use of a low-quality first-order 


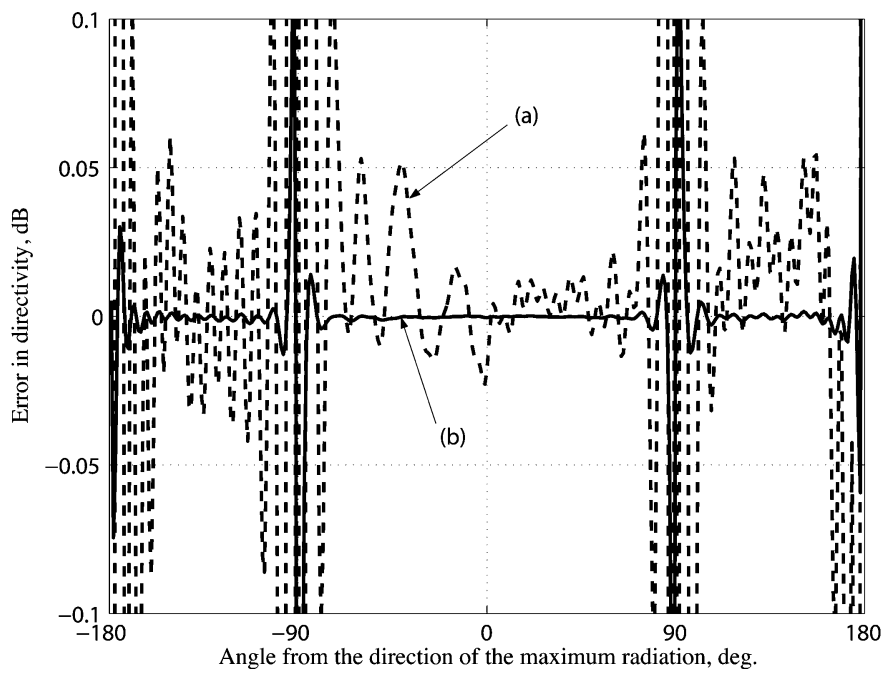

Fig. 3. Simulation result. The difference between the predicted and correct directivity of the AUT model in the $x-z$ plane (a) without iteration, i.e., using the traditional first-order probe correction, and (b) after one iteration. The nulls of the directivity in angles $\pm 90^{\circ}$ and $180^{\circ}$ explain the relatively high error level in those directions. probe for accurate spherical near-field antenna measurements. The cost of using the iterative technique compared with the existing first-order probe correction is a more time-consuming probe calibration and a slightly more time-consuming computation. It is important to note that the iterative technique has been observed to work, with certain restrictions, also for more general probes, e.g., for an open-ended rectangular waveguide. A more complete study on the applicability range of the technique will be presented elsewhere.

\section{REFERENCES}

[1] J. E. Hansen, Spherical Near-Field Antenna Measurements. London, U.K.: Peregrinus, 1988.

[2] T. A. Laitinen, J. M. Nielsen, S. Pivnenko, and O. Breinbjerg, "Errors of first-order probe correction for higher-order probes in spherical nearfield antenna measurements," in Proc. URSI Int. Symp. Electromagnetic Theory, Pisa, Italy, May 23-27, 2004, pp. 588-590.

[3] D. N. Black Jr. and E. B. Joy, "Test zone field compensation," IEEE Trans. Antennas Propag., vol. 43, no. 4, pp. 362-368, Apr. 1995.

[4] S. Pivnenko, J. M. Nielsen, and O. Breinbjerg, "Aspects of antenna pattern characterization of an L-band space radiometer," in Proc. AMTA'03, Irvine, CA, Oct. 19-24, 2003, pp. 375-379. 